\title{
Is there new physics around the corner?
}

C) ew results from the $\mathrm{LHCb}$ collaboration at CERN and the muon g- 2 collaboration at Fermilab have made headlines in recent weeks, not only in scientific journals but also in daily newspapers around the world, dubbing the standard model of particle physics getting cold feet or being at the end of the line. All lurid headlines aside, these new results make it clear that something new may be on the horizon.

\section{Discrepancies}

The LHCb result is putting stress on lepton universality between electrons and muons in specific decays of $\mathrm{B}$ mesons, of which the decay rates of $B^{+} \rightarrow K^{+} e^{+} e^{-}$and of $B^{+} \rightarrow K^{+} \mu^{+} \mu^{-}$are found to disagree at the 3.1 sigma level with each other. The muon $\mathrm{g}-2$ result is measuring a discrepancy at the 4.2 sigma level, where the measured value of the muon magnetic moment is off from its theoretically predicted value. Measuring the magnetic moment of electrons and comparing it with its theoretical prediction yields to the best tested result in physics, with more than 10 significant figures accuracy. Both discrepancies hint that muons and electrons behave differently, unexpectedly. Both these discrepancies are extremely tiny and it takes enormous effort to measure them.

\section{Evidence for new physics?}

Statistical fluctuations or a forgotten systematic effect may still be at the cause of these reported preliminary results. More data taking and more scrutiny is needed and will not lead to a final conclusion for another couple of years to come. Still, if both results could be confirmed, new particles would need to be added to the well-known quarks, leptons and bosons, where leptoquarks or supersymmetric particles are among the most promising candidates.

\section{Historical analog}

Changing from particle physics to gravity and changing time from now to the mid- $19^{\text {th }}$ century, another extremely tiny effect made history. Back then, measuring the perihelion of Mercury and how it shifts year after year has been done with great care over many decades. A shift that resulted to be off from its theoretical prediction by the extremely minute amount of only 43 arc seconds per century. The calculation, based on Newtonian gravity, included the gravitational pull of the sun and of the known planets. Adding a new planet to our solar system was proposed to provide remedy to this minute discrepancy. This hypothesized planet was dubbed Vulcan with an orbit inside the orbit of Mercury and so close to the sun that no optical instrument would be capable of seeing it directly. This was the state around mid 1850 and it took almost 70 years to resolve. The name Vulcan will ring a bell to all fans of the Star Trek series and of Mr Spock, the most prominent Vulcanian. Gene Roddenberry, the author of the original series, must have known about Vulcan and decided using it in his plot back in 1964. Einstein presented his theory of general relativity in 1915/16 and revolutionized the understanding of gravity. Conceptual flaws of the otherwise extremely successful Newtonian description of gravity were removed and the perihelion shift of Mercury be understood precisely without the need of Vulcan. Gravity is no longer a mysterious action at a distance, but is emerging from space-time itself, no longer acting instantaneously but propagating at the speed of light. Still, Newtonian gravity is being used for almost all calculations in everyday life. We do so in full confidence, because with the realm of general relativity, we know why Newtonian gravity works so well, and also up to which extreme conditions it will provide useful results. Newtonian gravity is, this way, fully complete.

\section{Future expectations}

We do not yet know what the new results from the $\mathrm{LHCb}$ and the muon g-2 collaborations tell us and where these will lead us. Maybe the hypothesised leptoquarks will have the same fate as the hypothesised planet Vulcan that, based on new insights obtained, was no longer needed. It took 70 years then, only the future will tell, how long it will take now. What is however clear already today, is that the Standard Model of particle physics works extremely well and will continue to be working extremely well. However, we do not yet know up to which extreme conditions it can provide useful results. The results from LHCb and muon g-2 may be those capable of paving the way further. 
Highlight your expertise. Get your company listed in europhysicsnews company directory For further information please contact bernadette.dufour@edpsciences.org

\begin{abstract}
BARTINGTON INSTRUMENTS www.bartington.com

Bartington Instruments designs and manufactures high precision fluxgate magnetometers, Helmholtz coil systems, gradiometers, magnetic susceptibility instruments and associated data processing equipment, for the precise generation and measurement of magnetic fields around physics experiments, and for calibration of magnetometers in consumer electronics.
\end{abstract}

\section{Bartington}

\section{${ }^{\circ}$ BLUEFORS}

\section{MCPHERSON}

MB SCIENTIFIC AB

www.mbscientific.se

MB Scientific AB is a Swedish company which develops and produces state of the art instruments for the photoelectron spectroscopy experiments. Our photoelectron energy analyser MBS A-1 gives you the opportunity to do world leading research together with MBSVUV photon sources, MBS L-1 and T-1, which produce the brightest and narrowest lines existing to be used for this type of experiments.

\section{$M B S$}

MB SCIENTIFIC AB

\section{BLUEFORS OY}

Bluefors.com

manufactures cryogenic measurement systems and has a strong Our in the field of quantum technology. can be customized to meet the special requirements of each experiment allowing the customer to get in direct contact with the scientists and engineers who design their system.

\section{WWW.mcphersoninc.com}

McPherson designs and manufactures scanning monochromators, flat-field imaging spectrographs, and vacuum monochromators and measurement systems for reflectance, transmittance, and absorbance testing. Its spectrometers and systems are built for soft x-ray, vacuum-ultraviolet, and UV/Vis and Infrared wavelengths. Applications range from lasers and lithography, solar, and energy to analytical life science and more.

\section{따바태네}

\section{PFEIFFER VACUUM}

\section{www.pfeiffer-vacuum.com/en/}

PfeifferVacuum stands for innovative and custom vacuum solutions worldwide, technological perfection, competent advice and reliable service. With the invention of the turbopump, the company paved the way for further development within the vacuum industry. Pfeiffer Vacuum offers a complete product portfolio: backing pumps, leak detectors, measurement and analysis devices, components as well as vacuum chambers and systems.

\section{PFEIFFER VACUUM}

\section{TOPTICA PHOTONICS}

\section{www.toptica.com}

TOPTICA Photonics, founded in 1998 near Munich (Germany), develops and manufactures high-end laser systems for scientific and industrial applications. The portfolio includes diode lasers, ultrafastfiber lasers, terahertz systems and frequency combs. OEM customers, scientists, and over a dozen Nobel laureates all acknowledge the world-class exceptional specifications ofTOPTICA's lasers, as well as their reliability and longevity.

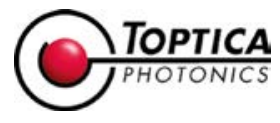

\section{INTERMODULATION PRODUCTS AB}

intermodulation-products.com

We develop and sell high-speed multichannel, multifrequency lock-in amplifiers with specialized signal processing capabilities for non-linear systems. In particular we have applications for determining the tip-surface force in tapping mode AFM, and an all-in-one measurement and control instrument for quantum computing.

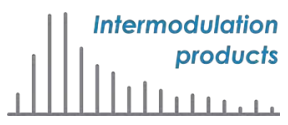

\section{OXFORD INSTRUMENTS}

\section{nanoscience.oxinst.com}

Oxford Instruments NanoScience provides market-leading cryogenic systems that enable quantum technologies, new materials and device development in the physical sciences. To learn more about Oxford Instruments NanoScience's low temperature systems for quantum computing applications, please visit our website.

\section{ZURICH INSTRUMENTS}

\section{www.zhinst.com}

Zurich Instruments is a technology leader developing and selling advanced test \& measurement instruments for dynamic signal analysis. These devices are used in many fields of application by hightechnology research laboratories and industrial development sites. Zurich Instruments' vision is to revolutionize instrumentation in the high-frequency and ultra-high-frequency -... range by incorporating the latest analog and digital technology into powerful measurement systems.
Zurich

Instruments 


\section{Cool for Progress.}

\section{BLUEFORS.COM}

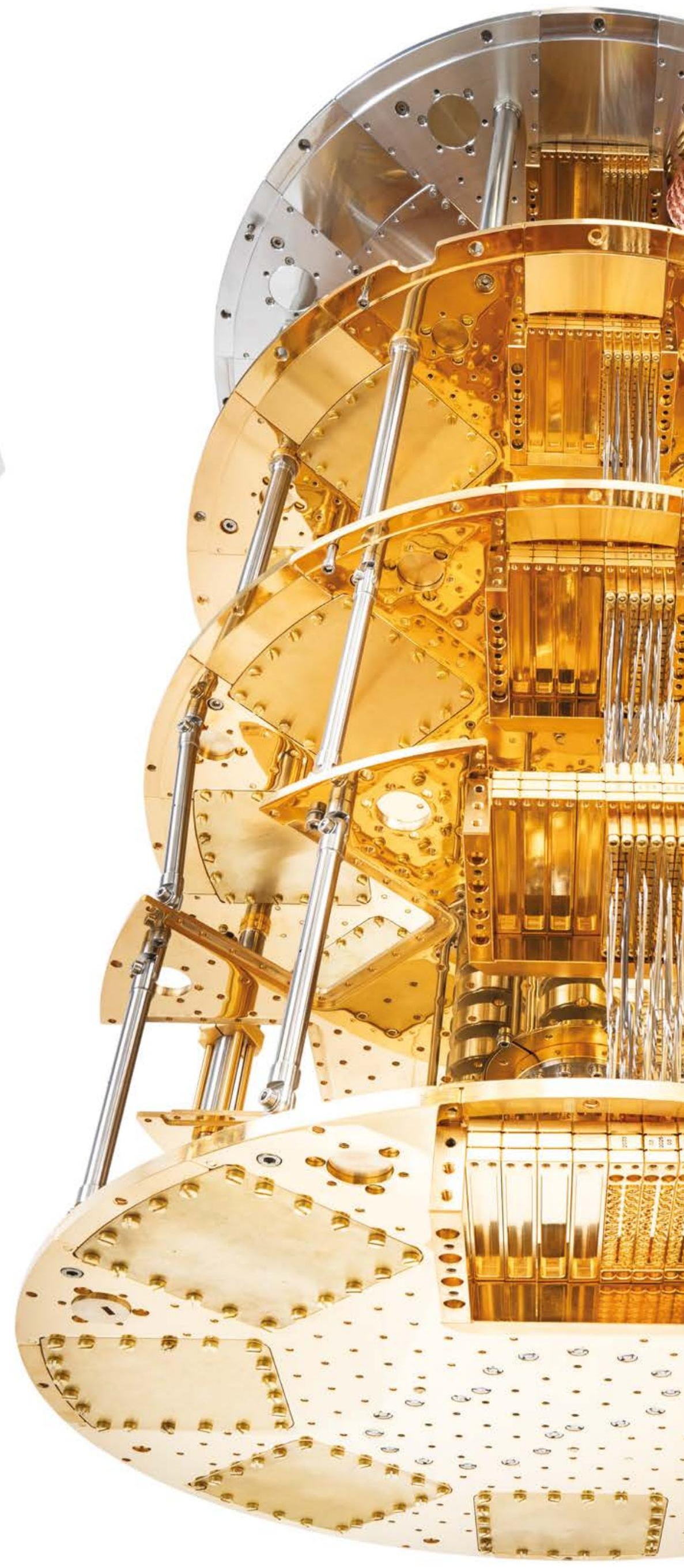

\section{High-density wiring}

Our new high-density wiring is a modular option for the Bluefors sideloading XLDsl dilution refrigerator system that enables a large scale-up of the experimental wiring, especially for high-frequency signals. It is easy to install and to maintain.

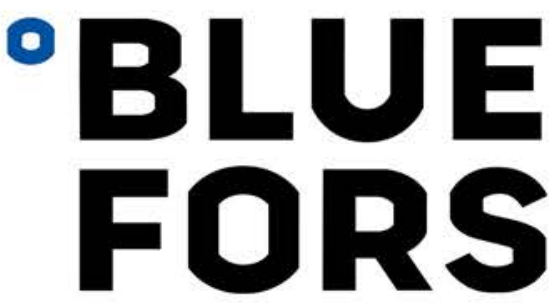

